\title{
Migration of Bone Wax into the Sigmoid Sinus after Posterior Fossa Surgery
}

\author{
(D) K. Byrns, (D) A. Khasgiwala, and (D). Patel
}

\begin{abstract}
BACKGROUND AND PURPOSE: Bone wax is a hemostatic agent that has been reported in some instances to migrate into the sigmoid sinus following posterior fossa surgery. The purpose of this study was to characterize the CT and MR imaging findings of this entity.

MATERIALS AND METHODS: The study included 212 consecutive patients who underwent posterior fossa surgery and postoperative CT and contrast-enhanced MR imaging. The presence of sigmoid sinus bone wax migration was determined with the following criteria: sigmoid sinus filling defect showing low signal on all MR imaging pulse sequences; sigmoid sinus filling defect showing low CT attenuation, similar to fat attenuation; and clinical confirmation that bone wax was used intraoperatively. CT and MR imaging of an in vitro bone wax sample was also performed.
\end{abstract}

RESULTS: We identified 6 cases of sigmoid sinus bone wax migration. In each case, a low-signal-intensity, low-attenuation filling defect was noted in the sigmoid sinus. The morphology was linear $(n=3)$ or globular $(n=3)$. In patients with serial imaging, the appearance of migrated bone wax remained stable over time. No adverse outcomes related to sigmoid sinus bone wax migration were encountered. In vitro imaging of bone wax confirmed low CT attenuation and low MR imaging signal intensity on TTWI and T2WI.

CONCLUSIONS: Bone wax migration into the sigmoid sinus is a recognizable imaging finding after posterior fossa surgery that appears to have a benign clinical course. The finding should be distinguished from more serious complications, such as venous sinus thrombosis.

ABBREVIATIONS: $\mathrm{HU}=$ Hounsfield units; $\mathrm{VIBE}=$ volumetric interpolated breath-hold examination

B one wax is commonly used during the course of posterior fossa craniotomy to control bleeding from emissary veins or to pack violated mastoid air cells. The mastoid emissary vein often lies close to or within the retrosigmoid or suboccipital craniotomy bed and represents a channel in direct communication with the sigmoid sinus. ${ }^{1}$ Awareness of the use of this agent is important in assessing postoperative imaging because its presence within a dural venous sinus might be mistaken for air, fat, or thrombus. The particular imaging characteristics of this agent can aid appropriate diagnosis. The appearance has been reported in the literature as low attenuation on CT (intermediate between fat and simple fluid) and as a signal void on MR imaging (attributable to its semicrystalline solid nature). ${ }^{2}$ To our knowledge, only 2 prior

Received March 4, 2016; accepted after revision May 15.

From the Department of Neuroradiology (K.B., A.K.), NYU Langone Medical Center, New York, New York; and Division of Neuroradiology (S.P.), University of Virginia Health System, Charlottesville, Virginia.

Please address correspondence to Kory A. Byrns, MD, Department of Neuroradiology, NYU Langone Medical Center, 660 1st Ave, New York, NY 10016; e-mail: kory.byrns@gmail.com

三 Indicates article with supplemental on-line table.

http://dx.doi.org/10.3174/ajnr.A4871 studies of sigmoid sinus bone wax migration (including 8 total cases) have been reported in the literature, and conventional MR imaging features of the migrated bone wax were reported in only 1 of these cases. ${ }^{3,4}$ The purpose of our study was to describe the $\mathrm{CT}$ and MR imaging features of sigmoid sinus bone wax migration after posterior fossa surgery. We also sought to assess the CT and MR imaging features of an in vitro bone wax sample.

\section{MATERIALS AND METHODS}

This Health Insurance Portability and Accountability Act-compliant retrospective study was reviewed and approved by our institutional review board.

To compile a set of patients who underwent posterior fossa surgery, a query of our radiology report data base was used to identify reports containing the terms "suboccipital," "retrosigmoid," or "mastoidectomy" generated over a 6-month period. Scans of the resultant patients were reviewed to identify those who had in fact undergone surgery. The patient list was further refined by including only those patients who underwent both CT and contrast-enhanced MR imaging postoperatively. A neuroradiologist with a Certificate of Added Qualification reviewed this subset of patients to identify cases of sigmoid sinus bone wax migration. 

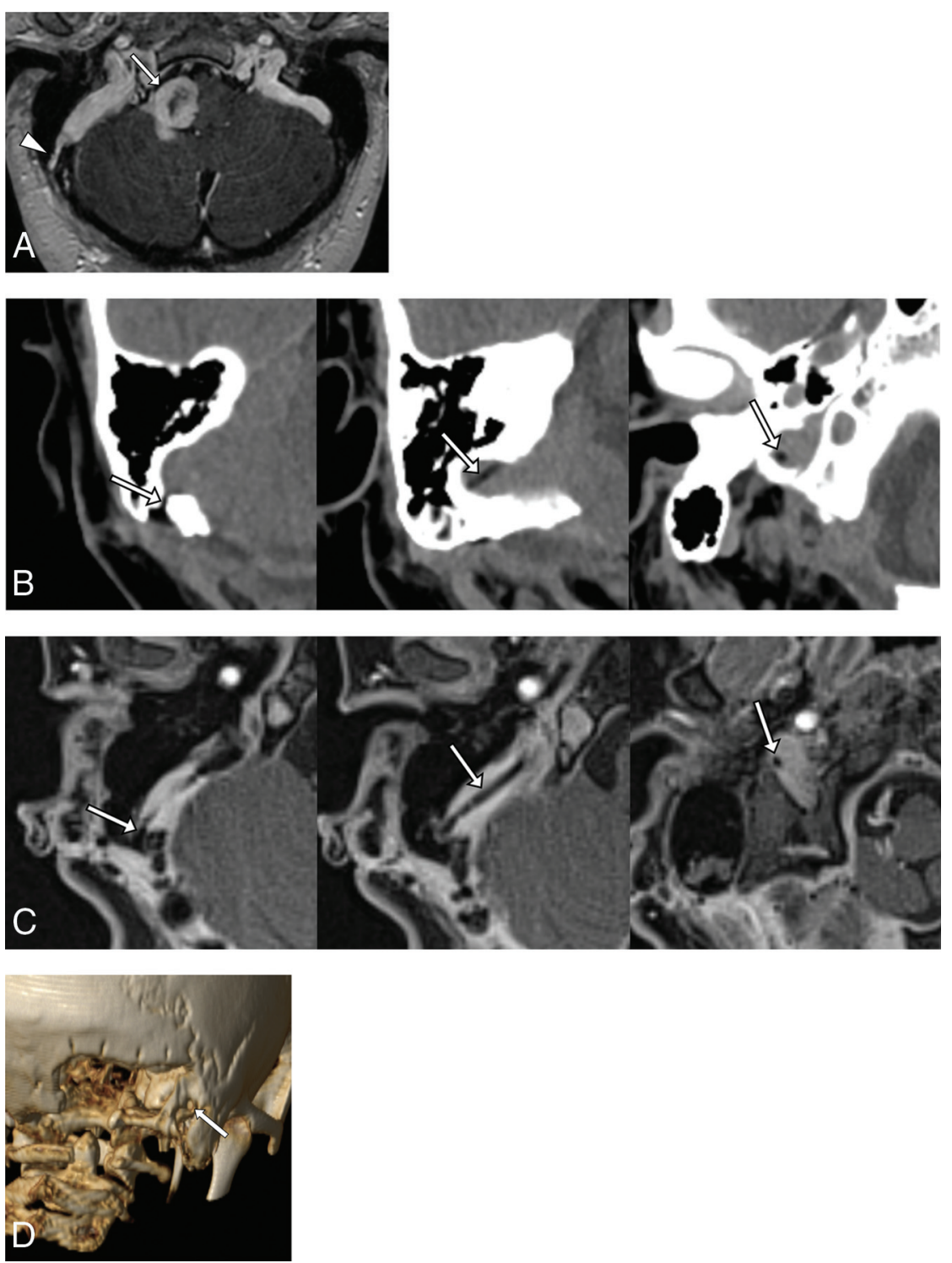

FIG 1. A, Axial contrast-enhanced TIWI demonstrates a lobulated enhancing lesion in the right perimedullary space exhibiting mass effect upon the medulla and right cerebellar hemisphere (arrow). A right mastoid emissary vein communicating with the sigmoid sinus is also visible (arrowhead). B, Sequential axial nonenhanced CT images obtained postoperatively demonstrate a linear low-attenuation $(-49 \mathrm{HU})$ stringlike structure traversing the mastoid canal and extending along the course of the sigmoid sinus to terminate at the level of the jugular foramen. $C$, Axial contrast-enhanced TIWI with fat suppression performed postoperatively demonstrates a linear low-signal filling defect within the sigmoid sinus following the course of the low-attenuation structure identified on CT. D, Volume-rendering technique of the $C T$ viewed posteriorly demonstrates the location of the foramen of the mastoid emissary vein just lateral to the craniectomy (arrow).

The criteria for sigmoid sinus bone wax migration were: 1) sigmoid sinus filling defect showing low signal intensity on all MR imaging pulse sequences; 2) sigmoid sinus filling defect showing low CT attenuation, similar to fat attenuation; and 3) confirmation via review of the medical record that bone wax was used intraoperatively. The demographic data, lesion treated, interval between surgery and imaging, and the morphology, location, and attenuation in Hounsfield units (HU) of the filling defect were recorded. All available postoperative imaging studies were reviewed to determine any change in the appearance of the migrated bone wax. Medical records of each patient were reviewed for any adverse clinical outcome related to bone wax migration.
The protocol used at our institution for postoperative MR imaging of the temporal bones and posterior fossa is as follows: whole brain sequences including axial T2WI (TR/TE 3800/116 ms, matrix $=320 \times 224$, section thickness $=$ $5 \mathrm{~mm})$, axial FLAIR $(\mathrm{TR} / \mathrm{TE} / \mathrm{TI}=9000 /$ $81 / 2500 \mathrm{~ms}$, matrix $=320 \times 224$, section thickness $=5 \mathrm{~mm})$, axial DWI (TR/ $\mathrm{TE}=7800 / 83 \mathrm{~ms} ; b=0,500,1000$; matrix $=150 \times 150$; section thickness $=$ $5 \mathrm{~mm}$ ), and postcontrast axial T1WI $(\mathrm{TR} / \mathrm{TE}=550 / 6.7 \mathrm{~ms}$, matrix $=320 \times$ 224 , section thickness $=5 \mathrm{~mm}$ ); precontrast temporal bone sequences including axial T1WI $(\mathrm{TR} / \mathrm{TE}=550 / 6.7 \mathrm{~ms}$, matrix $=320 \times 224$, section thickness $=3$ $\mathrm{mm})$ and axial CISS $(\mathrm{TR} / \mathrm{TE}=1000 / 136$ ms, matrix $=320 \times 317$, section thickness $=0.44-\mathrm{mm}$ ); and postcontrast temporal bone sequences including axial radial volumetric interpolated breath-hold examination (radial VIBE) $(\mathrm{TR} / \mathrm{TE}=$ $4.42 / 2.08 \mathrm{~ms}$, matrix $=224 \times 224$, section thickness $=0.8 \mathrm{~mm}$ ), axial T1WI with fat saturation $(\mathrm{TR} / \mathrm{TE}=550 / 6.7 \mathrm{~ms}$, matrix $=320 \times 224$, section thickness $=3$ $\mathrm{mm})$, and coronal T1WI (TR/TE $=538 /$ $6.7 \mathrm{~ms}$, matrix $=320 \times 224$, section thickness $=3 \mathrm{~mm}$ ). For several patients, a volumetric T1-weighted MPR sequence (TR/ TE/TI 2730/1000/3.31 ms, matrix = $256 \times 216$, section thickness $=1.10 \mathrm{~mm}$ ) was performed rather than the radial VIBE sequence. There was no intersection gap in any sequences performed.

In vitro imaging evaluation of bone wax obtained from our hospital's surgical supply was performed by using a $1-\mathrm{g}$ sample suspended within a $10-\mathrm{mL}$ plastic syringe containing sterile water. The sample was evaluated with CT and MR, including $\mathrm{T} 2$-weighted $(\mathrm{TR} / \mathrm{TE}=$ $3000 / 68 \mathrm{~ms}$, matrix $=256 \times 218$, section thickness $=2 \mathrm{~mm}$ ) and T1weighted $(\mathrm{TR} / \mathrm{TE}=450 / 11$, matrix $=$ $320 \times 282$, section thickness $=3 \mathrm{~mm}$ ) sequences. After adding a small amount of Gadavist (Bayer Schering Pharma, Berlin, Germany) gadolinium-based contrast to the syringe, an additional T1-weighted sequence $(\mathrm{TR} / \mathrm{TE}=613 / 11$, matrix $=320 \times 282$, section thickness $=3 \mathrm{~mm}$ ) was performed.

\section{RESULTS}

The initial data base query yielded 451 patients. Review of the available imaging for these patients yielded a total of 212 for whom surgery involving the posterior fossa had been performed and appropriate postoperative CT and MR imaging was available. Finally, review of this subset of patients yielded 6 patients who met 

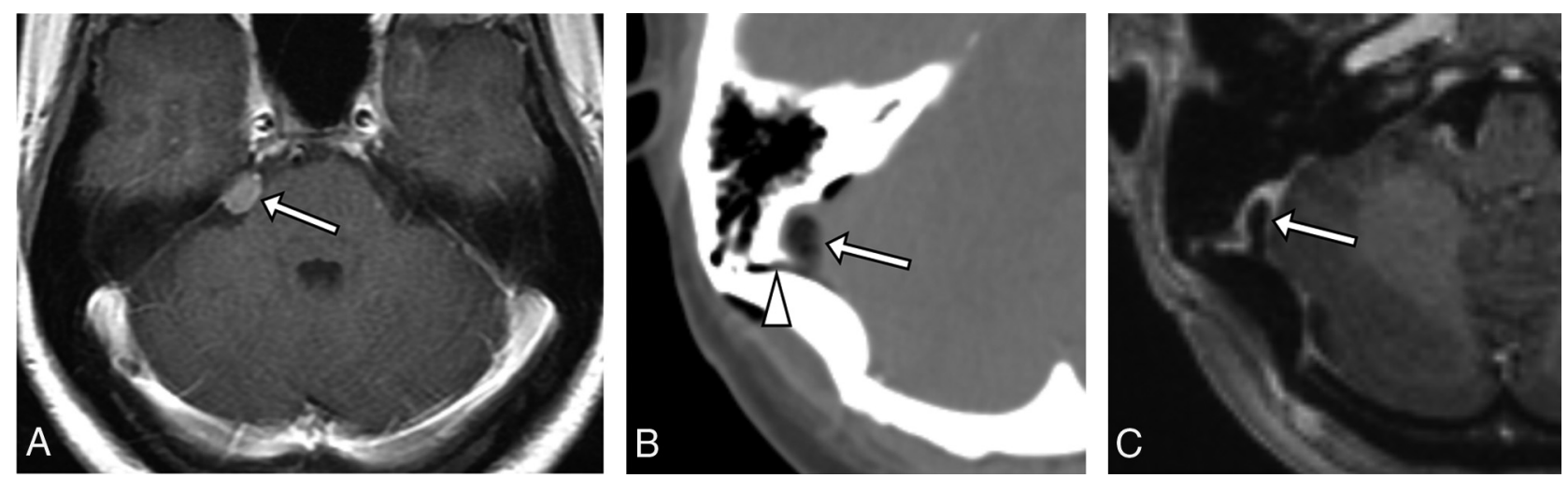

FIG 2. A, Axial contrast-enhanced TIWI demonstrates a small meningioma arising from the petrous ridge (arrow). B, Postoperative axial nonenhanced CT demonstrates a globular focus of low attenuation $(-162 \mathrm{HU}$ ) within the sigmoid sinus (arrow) adjacent to the craniotomy bed, which is noted to extend to the canal for the mastoid emissary vein (arrowhead). C, Postoperative axial contrast-enhanced TIWI shows that the filling defect is low signal (arrow), consistent with migrated bone wax. Note that the material is low signal even in the absence of fat-suppression techniques.

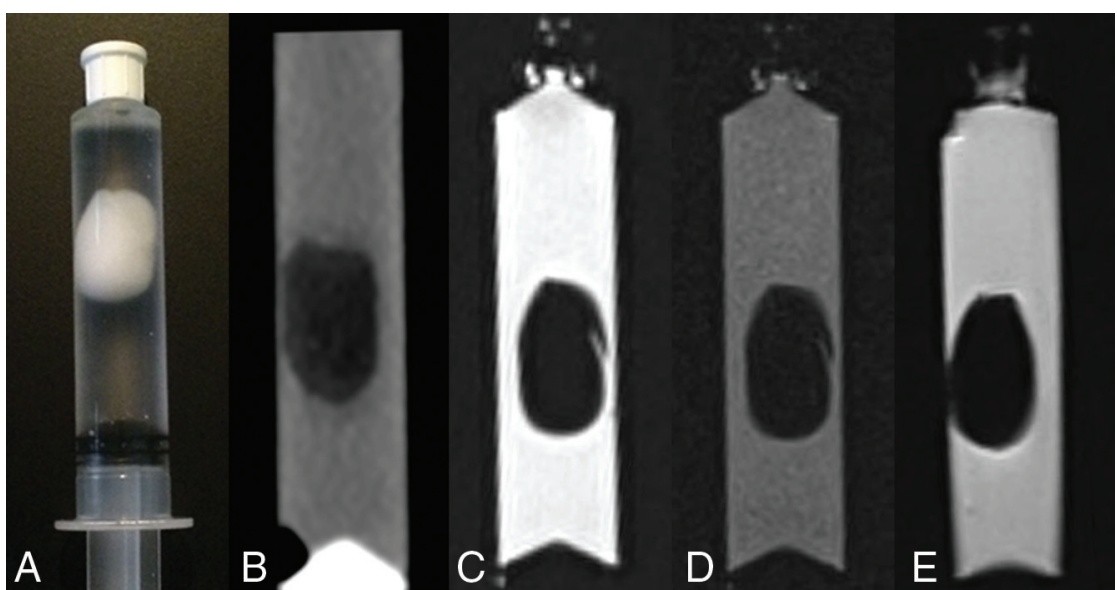

FIG 3. A, Photograph of a 1-g sample of bone wax suspended in sterile water within a 10-mL plastic syringe. $B, C T$ of the sample illustrates the low attenuation of the material $(-104 \mathrm{HU})$. There is uniformly low MR signal intensity on T2WI (C), TIWI (D), and TIWI performed after adding a small amount of gadolinium-based contrast to the solution $(E)$.

all criteria for a presumed diagnosis of migrated bone wax within the sigmoid sinus. A seventh patient met 2 of 3 criteria, though CT imaging was not available to confirm low attenuation of the filling defect that otherwise resembled migrated bone wax. A summary of our findings is presented in the On-line Table. For the 6 cases meeting all criteria, patient ages ranged from 30 to 78 years, and the group consisted of 5 women and 1 man. The lesions treated included a vestibular schwannoma, a spinal accessory nerve schwannoma, a clival meningioma, a petrous apex meningioma, a cerebellar hemangioblastoma, and an arachnoid cyst of the posterior fossa. The filling defects were nonocclusive and appeared globular in 3 cases and linear in the remaining 3. The material extended caudally into the jugular bulb or internal jugular vein in 3 of the cases. Measured attenuation ranged from -162 to -39 HU.

The interval between the initial operation and the most recent imaging varied from immediately postoperative to over 3 years, with a mean interval of 414 days. No appreciable change in the finding was observed for the cases with long-term follow-up available. Review of the medical records did not indicate any negative clinical outcome attributed to bone wax migration or reoperation performed for this reason. The interval between the patient's surgery and most recent clinical evaluation varied from 17 to 1213 days, with a mean interval of 475 days. One patient (case 3) presented 9 months postoperatively with seizures characteristic of known epilepsy. Imaging was stable at this time and no neurosurgical intervention was recommended. Another patient (case 5) presented 2 months postoperatively with drainage near the wound site, which was attributed to a small pustule, and no repeat imaging was recommended.

A representative case of linear migrated bone wax (case 3 ) is presented in Fig 1. Preoperative MR imaging demonstrates the excised lesion (a perimedullary schwannoma discovered to arise from the spinal accessory nerve) as well as a patent, well-developed mastoid emissary vein. Selected images after suboccipital craniotomy reveal a linear lowattenuation, low-signal filling defect traversing the emissary venous channel and coursing into the sigmoid sinus. Volumetric rendering of the $\mathrm{CT}$ demonstrates the proximity of the mastoid emissary foramen to the craniotomy site.

A case exemplifying globular migrated bone wax (case 4) is presented in Fig 2. Preoperative contrast-enhanced MR imaging demonstrates a small petrous ridge meningioma projecting into the cerebellopontine angle cistern. Immediate postoperative CT demonstrates a globular low-attenuation filling defect within the sigmoid sinus adjacent to the craniotomy bed, which extends into the nearby mastoid emissary vein channel. Contrast-enhanced MR performed 3 months later reveals a low-signal filling defect in a stable location and configuration.

Figure 3 shows a photograph of an in vitro sample of bone wax suspended in sterile water along with corresponding CT and MR images. The sample demonstrated low attenuation on CT $(-104$ $\mathrm{HU}$ ), and low signal intensity on T1WI and T2WI. 


\section{DISCUSSION}

The neurosurgical approach to deep posterior fossa lesions often necessitates extension of the craniotomy/craniectomy to include emissary venous foramina. In particular, the opening of the mastoid canal, which transmits the mastoid emissary vein, can be exposed or otherwise instrumented. The vein is an example of several variably present channels that act as an extracranial drainage conduit for the dural venous sinuses. This connection functions as an alternate pathway if the internal jugular vein is occluded or otherwise compromised. ${ }^{1}$

In the event of excessive venous bleeding encountered while instrumenting the region of the mastoid canal, a common method of achieving hemostasis is to apply bone wax. The bone wax used in our institution is a sterile mixture of beeswax, paraffin, and isopropyl palmitate (a wax-softening agent) that functions as a mechanical barrier to tamponade bleeding from divided, drilled, or chipped edges of bone. ${ }^{5}$ Bone wax is also used to seal off visible mastoid air cells after retrosigmoid craniotomy to prevent postoperative CSF leaks. ${ }^{6}$ This agent has remained in common use because it is inexpensive, biochemically inert, and nonabsorbable.

Reported complications related to bone wax application are varied but uncommon. These include granulomatous reaction, ${ }^{7-10}$ mass effect, ${ }^{2}$ saphenous vein graft thrombosis, ${ }^{11}$ epistaxis, ${ }^{12}$ calvarial osteohypertrophy, ${ }^{13}$ and infection. ${ }^{14}$ Retained, excessive, or migrated bone wax might simulate a mass. ${ }^{15}$ Bone wax has been used in the treatment of superior semicircular canal dehiscence and, in these cases, excessive wax application might migrate along the lumen to adversely affect the sensory neuroepithelium of the vestibular end organs. ${ }^{16}$ There is at least a theoretic risk of bone wax embolization when applied to the marrow space, as postulated in an animal study in which deposits were found in the lungs after application of radioactive bone wax to canine sternotomy incisions. ${ }^{17}$ Additional potential hazards noted by the manufacturer include inhibition of osteogenesis and the possibility of potentiating an infection, as with any foreign body. Instructions for use specifically warn that bone wax should be used sparingly and any excess should be removed from the operative site. ${ }^{5}$

Review of the neurosurgical literature revealed a single case series of migration of bone wax into the sigmoid sinus. Hadeishi et $\mathrm{al}^{3}$ reported 7 cases after retrosigmoid craniotomy from a series of 161 patients. Postoperative CT and time-of-flight MRV were reported in each case, though conventional MR imaging sequences were not described. Of the 7 reported patients, none demonstrated any sign of neurologic deterioration postoperatively. A single case report of symptomatic sigmoid sinus occlusion by bone wax was reported by Crocker et al. ${ }^{4}$ The patient developed acute exacerbation of headaches as well as transient hemiparesis on day 6 after retrosigmoid craniectomy for resection of a vestibular schwannoma. In this instance, the patient was anticoagulated and discharged on postoperative day 9 without further symptoms or additional surgical intervention. Of note, the imaging in this case revealed the lumen to be totally occluded by bone wax, with absence of flow in the ipsilateral transverse and sigmoid sinuses. In our series, the filling defects were uniformly nonocclusive, and review of medical records did not reveal any adverse outcome attributed to migrated bone wax.

There are several limitations of this investigation that should be noted. Pathologic confirmation of the diagnosis was not available because no additional surgical interventions were performed. The data base search method is inherently limited by variations in terminology and reporting. Thus, all instances of posterior fossa surgery that can lead to sigmoid sinus bone wax migration may not have been captured for the time period investigated. Moreover, we restricted our patient cohort to those who underwent both postoperative CT and MR imaging scans. Given these caveats, we are unable to report the true incidence of this finding after posterior fossa surgery.

\section{CONCLUSIONS}

The sigmoid sinus should be routinely inspected when interpreting postoperative imaging after postoperative craniotomy because thrombosis is a rare but reported complication of this approach. ${ }^{18,19}$ However, as illustrated by the cases presented here, detection of a filling defect should prompt careful evaluation of the attenuation and signal characteristics of the abnormality on all available modalities. The course, morphology, and extent of occlusion should be assessed, and consultation with the surgical team is suggested to determine what foreign materials were introduced into the surgical field. Migration of bone wax into the dural venous sinuses is a rare result of posterior fossa surgery that radiologists should be prepared to differentiate from more serious complications. The finding may persist for many years postoperatively and, as in our series, may not have any associated negative clinical outcome.

\section{REFERENCES}

1. Ginsberg L. The posterior condylar canal. AJNR Am J Neuroradiol 1994;15:969-72 Medline

2. Stein JM, Eskey CJ, Mamourian AC. Mass effect in the thoracic spine from remnant bone wax: an MR imaging pitfall. AJNR Am J Neuroradiol 2010;31:844-46 CrossRef Medline

3. Hadeishi H, Yasui N, Suzuki A. Mastoid canal and migrated bone wax in the sigmoid sinus: technical report. Neurosurgery 1995;36: 1220-23; discussion 1223-24 Medline

4. Crocker M, Nesbitt A, Rich P, et al. Symptomatic venous sinus thrombosis following bone wax application to emissary veins. Brit J Neurosurg 2008;22:798-800 CrossRef Medline

5. Bone Wax [package insert]. Bridgewater, New Jersey: Ethicon; 2015

6. Ling PY, Mendelson ZS, Reddy RK, et al. Reconstruction after retrosigmoid approaches using autologous fat graft-assisted Medpor Titan cranioplasty: assessment of postoperative cerebrospinal fluid leaks and headaches in 60 cases. Acta Neurochir (Wien) 2014;156: 1879-88 CrossRef Medline

7. Ateş $\mathrm{O}$, Cayli SR, Gürses I. Bone wax can cause foreign body granuloma in the medulla oblongata. Br J Neurosurg 2004;18:538-40 CrossRef Medline

8. Leprovost N, Taupin A, Soubeyrand E, et al. Foreign body granuloma due to Horsley wax [in French]. Rev Stomatol Chir Maxillofac 2011;112:117-20 CrossRef Medline

9. Low WK, Sim CS. Bone wax foreign body granuloma in the mastoid. ORL J Otorhinolaryngol Relat Spec 2002;64:38-40 CrossRef Medline

10. Wolvius EB, van der Wal KG. Bone wax as a cause of a foreign body granuloma in a cranial defect: a case report. Int J Oral Maxillofacial Surgery 2003;32:656-58 CrossRef Medline

11. Chun PK, Virmani R, Mason TE, et al. Bone wax granuloma causing 
saphenous vein graft thrombosis. Am Heart J 1988;115:1310-13 CrossRef Medline

12. Tay HL, Tan LK. Surgical bone wax causing epistaxis. J Laryngol Otol 1996;110:267-68 Medline

13. Kamide T, Nakada M, Hirota Y, et al. Skull osteohypertrophy as a complication of bone wax. J Clin Neuroscience 2009;16:1658-60 CrossRef

14. Gibbs L, Kakis A, Weinstein P, et al. Bone wax as a risk factor for surgical-site infection following neurospinal surgery. Infect Control Hosp Epidemiol 2004;25:346-48 CrossRef Medline

15. Karabekır HS, Korkmaz S. Residue bone wax simulating spinal tumour: a case report. Turk Neurosurg 2010;20:524-26 CrossRef Medline

16. Cheng YS, Kozin ED, Remenschneider AK, et al. Characteristics of wax occlusion in the surgical repair of superior canal dehiscence in human temporal bone specimens. Otol Neurotol 2016;37:83-88 CrossRef Medline

17. Robicsek F, Masters TN, Littman L, et al. The embolization of bone wax from sternotomy incisions. Annals Thorac Surg 1981;31:357-59 CrossRef

18. Keiper GL Jr., Sherman JD, Tomsick TA, et al. Dural sinus thrombosis and pseudotumor cerebri: unexpected complications of suboccipital craniotomy and translabyrinthine craniectomy. J Neurosurg 1999;91:192-97 CrossRef Medline

19. Lega BC, Yoshor D. Postoperative dural sinus thrombosis in a patient in a hypercoagulable state. Case report. J Neurosurg 2006;105: 772-74 CrossRef Medline 